\title{
Differentiability of Spatially Homogeneous Solutions of the Boltzmann Equation in the Non Maxwellian Case
}

\author{
Gabriella Di Blasio \\ Istituto Matematico, Università degli Studi, Roma, Italy
}

Received February 23, 1974

\begin{abstract}
The non linear Boltzmann equation is studied and differentiable solutions are shown to exist if the initial datum is suitably chosen

\section{Introduction}

In the present paper we study the initial value problem for the Boltzmann equation in the spatially homogeneous case and obtain an existence and uniqueness theorem which is a generalization of [4] when the extrinsic force is zero.

This space-independent problem has been much studied; for instance in [9] and [1] Povzner and Bodmer obtain a similar result by completely different methods. We note however that our methods are much more simple; moreover Povzner does not investigate the differentiability of the solution while Bodmer considers a somewhat less general equation.
\end{abstract}

\section{Preliminaries}

We begin with some basic definitions and results which will be used in the rest of this paper.

Let $X$ be a real Banach space and $X^{*}$ its dual; by \|\| we denote the norm in $X$ and by $\left\langle x, x^{*}\right\rangle$ the value of $x^{*} \in X^{*}$ at $x \in X$.

With each $x \in X$ is associated the set $\partial\|x\|=\left\{x^{*} \in X^{*},\|x+y\|\right.$ $\left.\geqq\|x\|+\left\langle y, x^{*}\right\rangle, \forall y \in X\right\}$; the application $X \rightarrow 2^{X^{*}}, x \rightarrow \partial\|x\|$, is called the subdifferential of the norm.

Let $f: D_{f} \subset X \rightarrow X$ and consider the initial value problem

$$
\left\{\begin{array}{l}
\frac{d u}{d t}=f(u) \quad t \in[0, T] . \\
u(0)=u_{0}
\end{array}\right.
$$

A continuous function $u:[0, T] \rightarrow X$ is called a solution of (1) if it is differentiable in $[0, T]$ and satisfies (1). 
We will use the following lemma (see [7])

Lemma 1. Let $u$ belong to $C^{1}(0, T ; X)^{1}$; then $\|u(\cdot)\|$ is differentiable a.e. in $[0, T]$ and we have

$$
\frac{d}{d t}\|u(t)\|=\left\langle\frac{d u}{d t}, u^{*}\right\rangle, \quad \forall u^{*} \in \partial\|u(t)\| .
$$

\section{Properties of the Boltzmann Equation}

We shall study the Boltzmann equation in the spatially homogeneous case

$$
\frac{\partial u(\xi, t)}{\partial t}=f(u, u), \quad \xi \in \mathbb{R}^{3}, t \geqq 0 .
$$

The bilinear function $(u, v) \rightarrow f(u, v)$ is defined as follows ${ }^{2}$

where

$$
f(u, v)=f^{+}(u, v)-f^{-}(u, v)
$$

and

$$
f^{+}(u, v)=\frac{1}{2} \int_{\mathbb{R}^{3}} \int_{0}^{2 \pi} \int_{0}^{\pi / 2}\left[u\left(\xi^{\prime}\right) v\left(\xi_{1}^{\prime}\right)+u\left(\xi_{1}^{\prime}\right) v\left(\xi^{\prime}\right)\right] B\left(\theta, \xi, \xi_{1}\right) d \theta d \sigma d \xi_{1}
$$

$$
f^{-}(u, v)=\frac{1}{2} \int_{\mathbb{R}^{3}} \int_{0}^{2 \pi} \int_{0}^{\pi / 2}\left[u(\xi) v\left(\xi_{1}\right)+u\left(\xi_{1}\right) v(\xi)\right] B\left(\theta, \xi, \xi_{1}\right) d \theta d \sigma d \xi_{1}
$$

(usually the time dependence of $u$ and $v$ will not be written explicitly).

Here the variables $\xi^{\prime}, \xi_{1}^{\prime}$ depend on $\xi, \xi_{1}$ through

$$
\begin{gathered}
\xi^{\prime}=\xi+\alpha\left(\alpha \cdot\left(\xi-\xi_{1}\right)\right), \\
\xi_{1}^{\prime}=\xi_{1}-\alpha\left(\alpha \cdot\left(\xi-\xi_{1}\right)\right),
\end{gathered}
$$

where $\alpha=(\cos \theta, \sin \theta \cos \sigma, \sin \theta \sin \sigma)$. For fixed $\alpha$ these formulae represent a unitary transformation in the 6 -space $\xi, \xi_{1}$ so giving

$$
\partial\left(\xi^{\prime}, \xi_{1}^{\prime}\right) / \partial\left(\xi, \xi_{1}\right)=1 \text {. }
$$

Equations (2) mean in particular that

$$
\begin{gathered}
\xi+\xi_{1}=\xi^{\prime}+\xi_{1}^{\prime}, \\
\xi^{2}+\xi_{1}^{2}=\xi^{\prime 2}+\xi_{1}^{\prime 2}
\end{gathered}
$$

$B\left(\theta, \xi, \xi_{1}\right):(0, \pi / 2) x \mathbb{R}^{6} \rightarrow \mathbb{R}$ is a given measurable function non negative a.e. such that

$$
\begin{aligned}
& B\left(\theta, \xi, \xi_{1}\right)=B\left(\theta, \xi_{1}, \xi\right), \\
& B\left(\theta, \xi, \xi_{1}\right)=B\left(\theta, \xi^{\prime}, \xi_{1}^{\prime}\right) .
\end{aligned}
$$

We recall that owing to (3), (4), and (5) $f$ satisfies the following equalities ${ }^{3}$

$$
\int f(u, v) d \xi=0, \quad \int \xi^{2} f(u, v) d \xi=0
$$

${ }^{1} C^{m}(0, T ; X)$ is the Banach space of the $m$-times continuously differentiable functions $u:[0, T] \rightarrow X$ equipped with the norm $\|u\|=\sup _{t \in[0, T]}\|u(t)\|+\cdots+\sup _{t \in[0, T]}\left\|\frac{d^{m}}{d t^{m}} u(t)\right\|$.

2 Troughout this section by $u$ and $v$ we denote measurable functions.

3 For a more detailed description and proofs of the properties quoted in this section see [2], [6]. 
where $u$ and $v$ are any functions such that $f(u, v)$ exists. From now on we assume that the following hypothesis is fulfilled

$$
\text { (H) } \sup _{\xi, \xi_{1} \in \mathbb{R}^{3}} \int_{0}^{\pi / 2} \frac{B\left(\theta, \xi, \xi_{1}\right)}{1+\sqrt{\xi^{2}+\xi_{1}^{2}}} d \theta=B_{0}<+\infty
$$

An immediate consequence of $(\mathrm{H})$ is the following lemma.

Lemma 2. We have

$$
\int|f(u, v)| d \xi \leqq \gamma \int\left(1+\xi^{2}\right)|u| d \xi \int\left(1+\xi^{2}\right)|v| d \xi,
$$

where $\gamma=8 \pi B_{0}$.

We now want to establish some basic properties of $f$ which will be used in the following.

Lemma 3. Let $u$ be non negative a.e. We have

$$
\int\left(1+\xi^{2}\right)^{2} f(u, u) d \xi \leqq \gamma \int\left(1+\xi^{2}\right)^{2} u d \xi \int\left(1+\xi^{2}\right) u d \xi
$$

Proof. Set $B^{\prime}\left(\theta, \xi, \xi_{1}\right)=B\left(\theta, \xi, \xi_{1}\right)\left(\xi^{2}+\xi_{1}^{2}\right)$; it follows from (4) that $B^{\prime}$ satisfies (5), then (6) is verified

$$
\int \xi^{2} \int\left[u\left(\xi^{\prime}\right) u\left(\xi_{1}^{\prime}\right)-u(\xi) u\left(\xi_{1}\right)\right] B^{\prime}\left(\theta, \xi, \xi_{1}\right) d \theta d \sigma d \xi_{1} d \xi=0
$$

(the limits of integration have been omitted for simplicity in notation) and we have

$\int \xi^{4} f(u, u) d \xi=-\int \xi^{2} \int \xi_{1}^{2}\left[u\left(\xi^{\prime}\right) u\left(\xi_{1}^{\prime}\right)-u(\xi) u\left(\xi_{1}\right)\right] B\left(\theta, \xi, \xi_{1}\right) d \theta d \sigma d \xi_{1} d \xi$.

Now (6) implies

$$
\begin{aligned}
\int\left(1+\xi^{2}\right)^{2} f(u, u) d \xi= & \int \xi^{4} f(u, u) d \xi \\
= & \int \xi^{2} \int \xi_{1}^{2}\left[u(\xi) u\left(\xi_{1}\right)-u\left(\xi^{\prime}\right) u\left(\xi_{1}^{\prime}\right)\right] B\left(\theta, \xi, \xi_{1}\right) \\
& \cdot d \theta d \sigma d \xi_{1} d \xi \\
= & \int\left(1+\xi^{2}\right) \int\left(1+\xi_{1}^{2}\right)\left[u(\xi) u\left(\xi_{1}\right)-u\left(\xi^{\prime}\right) u\left(\xi_{1}^{\prime}\right)\right] \\
& B\left(\theta, \xi_{1}, \xi_{1}\right) d \theta d \sigma d \xi_{1} d \xi \\
\leqq & \int\left(1+\xi^{2}\right) u(\xi) \int\left(1+\xi_{1}^{2}\right) u\left(\xi_{1}\right) B\left(\theta, \xi, \xi_{1}\right) d \theta d \sigma d \xi_{1} d \xi
\end{aligned}
$$

and the conclusion follows from $(\mathrm{H})$.

Lemma 4. If $u$ and $v$ are any functions non negative a.e. then ${ }^{4}$

$$
\begin{array}{r}
\int\left(1+\xi^{2}\right) \operatorname{sgn}(u-v)[f(u, u)-f(v, v)] d \xi \leqq 2 \gamma \int\left(1+\xi^{2}\right)^{2}(u+v) d \xi \\
\int\left(1+\xi^{2}\right)|u-v| d \xi .
\end{array}
$$

${ }^{4} \operatorname{sgn} u$ is the function defined as follows

$$
\operatorname{sgn} u \begin{cases}=1 & \text { if } \quad u>0 \\ =-1 & \text { if } \quad u<0 \\ =0 & \text { elsewhere }\end{cases}
$$


Proof. We have

$$
\begin{aligned}
& \int\left(1+\xi^{2}\right) \operatorname{sgn}(u-v)[f(u, u)-f(v, v)] d \xi \\
& \leqq \int\left(1+\xi^{2}\right) \int\left|u\left(\xi_{1}\right)-v\left(\xi_{1}\right)\right|[u(\xi)+v(\xi)] B\left(\theta, \xi, \xi_{1}\right) d \theta d \sigma d \xi_{1} d \xi \\
&-\int\left(1+\xi^{2}\right) \int|u(\xi)-v(\xi)|\left[u\left(\xi_{1}\right)+v\left(\xi_{1}\right)\right] B\left(\theta, \xi, \xi_{1}\right) d \theta d \sigma d \xi_{1} d \xi \\
&+\int\left(1+\xi^{2}\right) \int\left|u\left(\xi^{\prime}\right)-v\left(\xi^{\prime}\right)\right|\left[u\left(\xi_{1}^{\prime}\right)+v\left(\xi_{1}^{\prime}\right)\right] B\left(\theta, \xi_{1}, \xi_{1}\right) d \theta d \sigma d \xi_{1} d \xi \\
&+\int\left(1+\xi^{2}\right) \int\left|u\left(\xi_{1}^{\prime}\right)-v\left(\xi_{1}^{\prime}\right)\right|\left[u\left(\xi^{\prime}\right)+v\left(\xi^{\prime}\right)\right] B\left(\theta, \xi_{1}, \xi_{1}\right) d \theta d \sigma d \xi_{1} d \xi \\
&= 2 \int\left(1+\xi^{2}\right)[u(\xi)+v(\xi)] \int\left|u\left(\xi_{1}\right)-v\left(\xi_{1}\right)\right| B\left(\theta, \xi, \xi_{1}\right) d \theta d \sigma d \xi_{1} d \xi
\end{aligned}
$$

where we used (6). Thus the conclusion follows from $(\mathrm{H})$.

For each $\varepsilon \in[0,1]$ let $f_{\varepsilon}$ be the bilinear function $(u, v) \rightarrow f_{\varepsilon}(u, v)$ $=f_{\varepsilon}^{+}(u, v)-f_{\varepsilon}^{-}(u, v)$, where and

$$
f_{\varepsilon}^{+}(u, v)=\frac{1}{2} \int\left[u\left(\xi^{\prime}\right) v\left(\xi_{1}^{\prime}\right)+u\left(\xi_{1}^{\prime}\right) v\left(\xi^{\prime}\right)\right] \frac{B\left(\theta, \xi, \xi_{1}\right)}{1+\varepsilon \sqrt{\xi^{2}+\xi_{1}^{2}}} d \theta d \sigma d \xi_{1}
$$

$$
f_{\varepsilon}^{-}(u, v)=\frac{1}{2} \int\left[u(\xi) v\left(\xi_{1}\right)+u\left(\xi_{1}\right) v(\xi)\right] \frac{B\left(\theta, \xi, \xi_{1}\right)}{1+\varepsilon \sqrt{\xi^{2}+\xi_{1}^{2}}} d \theta d \sigma d \xi_{1}
$$

By definition $f_{0}(u, v)=f(u, v)$; moreover as $B_{\varepsilon}\left(\theta, \xi, \xi_{1}\right)=B\left(\theta, \xi, \xi_{1}\right)$ $\left(1+\varepsilon \sqrt{\xi^{2}+\xi_{1}^{2}}\right)^{-1}$ satisfies (5) we have that each $f_{\varepsilon}$ verifies $(6),(7)$, and (8).

The following lemma establishes further properties of $f_{\varepsilon}$.

Lemma 5. For each $\varepsilon, \eta \in[0,1]$ the following inequalities hold:

$$
\int\left|f_{\varepsilon}(u, u)-f_{\eta}(u, u)\right| d \xi \leqq 4 \gamma\left(\int\left(1+\xi^{2}\right)|u| d \xi\right)^{2}|\varepsilon-\eta|
$$

$\int\left(1+\xi^{2}\right)\left|f_{\varepsilon}(u, u)-f_{\eta}(u, u)\right| d \xi \leqq 4 \gamma|\varepsilon-\eta| \int\left(1+\xi^{2}\right)^{2}|u| d \xi \int\left(1+\xi^{2}\right)|u| d \xi$.

Proof. We have

$$
\begin{aligned}
& \int\left|f_{\varepsilon}(u, u)-f_{\eta}(u, u)\right| d \xi \\
& \quad=\int\left|\int\left[u\left(\xi^{\prime}\right) u\left(\xi_{1}^{\prime}\right)-u(\xi) u\left(\xi_{1}\right)\right]\left[B_{\varepsilon}\left(\theta, \xi_{1}, \xi_{1}\right)-B_{\eta}\left(\theta, \xi, \xi_{1}\right)\right] d \theta d \sigma d \xi_{1}\right| d \xi \\
& \quad \leqq \iint\left[\left|u\left(\xi^{\prime}\right) u\left(\xi_{1}^{\prime}\right)\right|+\left|u(\xi) u\left(\xi_{1}\right)\right|\right]\left|B_{\varepsilon}\left(\theta, \xi_{1}, \xi_{1}\right)-B_{\eta}\left(\theta, \xi, \xi_{1}\right)\right| d \theta d \sigma d \xi_{1} d \xi \\
& \quad=2 \iint\left|u(\xi) u\left(\xi_{1}\right)\right|\left|\frac{(\varepsilon-\eta) \sqrt{\xi^{2}+\xi_{1}^{2}} B\left(\theta, \xi_{1}\right)}{\left(1+\varepsilon \sqrt{\xi^{2}+\xi_{1}^{2}}\right)\left(1+\eta \sqrt{\xi^{2}+\xi_{1}^{2}}\right)}\right| d \theta d \sigma d \xi_{1} d \xi \\
& \quad \leqq|\varepsilon-\eta| 4 \pi B_{0} \int|u(\xi)| \int\left|u\left(\xi_{1}\right)\right|\left(\sqrt{1+\xi^{2}}+\sqrt{1+\xi_{1}^{2}}\right)^{2} d \xi_{1} d \xi \\
& \quad \leqq|\varepsilon-\eta| 16 \pi B_{0} \int\left(1+\xi^{2}\right)|u(\xi)| d \xi \int\left(1+\xi_{1}^{2}\right)\left|u\left(\xi_{1}\right)\right| d \xi_{1},
\end{aligned}
$$

where we used (6) and (H). The second inequality follows in the same way. 
We will be concerned with the following Cauchy problem

$$
\text { (I) }\left\{\begin{aligned}
\frac{\partial u(\xi, t)}{d t} & =f(u, u) \\
u(\xi, 0) & =u_{0}(\xi)
\end{aligned}\right.
$$

and will prove that, under proper assumptions on the initial datum (I) has a unique solution.

\section{The Approaching Problem}

Let us denote by $X_{r}(r=0,1,2)$ the Banach space of the measurable functions $u: \mathbb{R}^{3} \rightarrow \mathbb{R}$ such that

$$
\|u\|_{r}=\int\left(1+\xi^{2}\right)^{r}|u| d \xi<+\infty
$$

equipped with the norm \|\|$_{r}$ and by $X_{r}^{*}$ its dual. By $\partial \|_{r}$ we denote the subdifferential of the norm in $X_{r}(r=0,1,2)$ and by $\langle,\rangle_{r}: X_{r} \times X_{r}^{*} \rightarrow \mathbb{R}$ the function $\left(u, u^{*}\right) \rightarrow\left\langle u, u^{*}\right\rangle_{r}=\int\left(1+\xi^{2}\right)^{r} u u^{*} d \xi$.

Let $Q$ be the closed convex cone $Q=\left\{u \in X_{0}, u \geqq 0\right.$ a.e. $\}$. For each $\varepsilon \in(0,1]$ we denote by $f_{\varepsilon}$ the function $f_{\varepsilon}: Q \rightarrow X_{0}, u \rightarrow f_{\varepsilon}(u)=f_{\varepsilon}(u, u)$ and by $f_{0}$ the function $f_{0}: Q \cap X_{1} \rightarrow X_{0}, u \rightarrow f_{0}(u)=f(u, u)$.

We prove the lemma.

Lemma 6. Let $u \in X_{r}$ then $\operatorname{sgn} u \in \partial\|u\|_{r},(r=0,1,2)$.

Proof. It is known that $\operatorname{sgn} u \in X_{r}^{*}$ (see [5]); moreover for each $v \in X_{r}$ we have $\|u+v\|_{r}=\langle u+v, \operatorname{sgn}(u+v)\rangle_{r} \geqq\langle u+v, \operatorname{sgn} u\rangle_{r}=\|u\|_{r}$ $+\langle v, \operatorname{sgn} u\rangle_{r}$.

The following lemmas follow from $(\mathrm{H}),(6)$ and Lemmas 3,4 and 5.

Lemma 7. We have

(i) if $u \in Q \cap X_{1}$ then for each $\varepsilon \in(0,1] f_{\varepsilon}(u) \in X_{1}$ and $\left\langle f_{\varepsilon}(u) \text {, sgn } u\right\rangle_{1}=0$,

(ii) if $u \in Q \cap X_{2}$ then for each $\varepsilon \in(0,1] f_{\varepsilon}(u) \in X_{2}$ and $\left\langle f_{\varepsilon}(u) \text {, sgn } u\right\rangle_{2}$ $\leqq \gamma\|u\|_{1}\|u\|_{2}$,

(iii) if $u, v \in Q \cap X_{2}$ then for each $\varepsilon \in[0,1] f_{\varepsilon}(u), f_{\varepsilon}(v) \in X_{1}$ and $\left\langle f_{\varepsilon}(u)-f_{\varepsilon}(v), \operatorname{sgn}(u-v)\right\rangle_{1} \leqq 2 \gamma\|u+v\|_{2}\|u-v\|_{1}$.

Lemma 8. For each $\varepsilon, \eta \in[0,1]$ we have

$$
\begin{array}{lll}
\text { (i) }\left\|f_{\varepsilon}(u)-f_{\eta}(u)\right\|_{0} \leqq 4 \gamma|\varepsilon-\eta|\|u\|_{1}^{2}, & \forall u \in Q \cap X_{1}, \\
\text { (ii) }\left\|f_{\varepsilon}(u)-f_{\eta}(u)\right\|_{1} \leqq 4 \gamma|\varepsilon-\eta|\|u\|_{1}\|u\|_{2}, & \forall u \in Q \cap X_{2} .
\end{array}
$$

Lemma 9. For each $u, v \in Q \cap X_{1}$ we have $f_{0}(u), f_{0}(v) \in X_{0}$ and

$$
\left\|f_{0}(u)-f_{0}(v)\right\|_{0} \leqq \gamma\|u-v\|_{1} .
$$


Let us consider the initial value problem

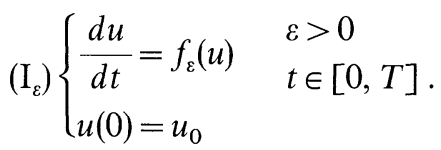

In [4] we proved the following theorem ${ }^{5}$ :

Theorem I. Assume that $u_{0} \in Q$. Then for each $T>0$ there exists a unique $u \in C^{1}(0, T ; X)$ solution of $\left(\mathrm{I}_{\varepsilon}\right)$; moreover for each $t \in[0, T]$ we have $u(t) \in Q,\|u(t)\|_{0}=\left\|u_{0}\right\|_{0}$.

The following theorem establishes further properties of the solution of $\left(I_{\varepsilon}\right)$ :

Theorem II. Let $u_{0} \in Q \cap X_{2}$ and let $u_{\varepsilon}$ be the solution of $\left(\mathrm{I}_{\varepsilon}\right)$; for each $t \in[0, T]$ we have:

$$
\begin{aligned}
& \left\|u_{\varepsilon}(t)\right\|_{1}=\left\|u_{0}\right\|_{1} \\
& \left\|u_{\varepsilon}(t)\right\|_{2} \leqq e^{\gamma\left\|u_{0}\right\|_{1} t}\left\|u_{0}\right\|_{2} .
\end{aligned}
$$

Proof. (i) and (ii) are an immediate consequence of (i) and (ii) of Lemma 7.

\section{Existence of the Differentiable Solution}

In this section we will investigate the existence of the solution of the following Cauchy problem

$$
\left(\mathrm{I}_{0}\right)\left\{\begin{array}{l}
\frac{d u}{d t}=f_{0}(u) \\
u(0)=u_{0},
\end{array} \quad t \in[0, T] .\right.
$$

We begin with two lemmas.

Lemma 10. Let $u_{0} \in Q \cap X_{2}$ and let $u_{\varepsilon}$ be the solution of $\left(I_{\varepsilon}\right)$; then there exists $u \in C\left(0, T ; X_{1}\right)$ such that $\lim _{\varepsilon \rightarrow 0} u_{\varepsilon}=u$ in $C\left(0, T ; X_{1}\right)$.

Proof. It follows from Lemma 1 that for each $\varepsilon, \eta \in(0,1]$ and a.e. $t \in[0, T]$ we have

$$
\begin{aligned}
\frac{d}{d t} \| u_{\varepsilon}(t) & -u_{\eta}(t) \|_{1}=\left\langle\frac{d}{d t} u_{\varepsilon}-\frac{d}{d t} u_{\eta}, \operatorname{sgn}\left(u_{\varepsilon}-u_{\eta}\right)\right\rangle_{1} \\
& =\left\langle f_{\varepsilon}\left(u_{\varepsilon}\right)-f_{\varepsilon}\left(u_{\eta}\right), \operatorname{sgn}\left(u_{\varepsilon}-u_{\eta}\right)\right\rangle_{1}+\left\langle f_{\varepsilon}\left(u_{\eta}\right)-f_{\eta}\left(u_{\eta}\right), \operatorname{sgn}\left(u_{\varepsilon}-u_{\eta}\right)\right\rangle_{1} \\
& \leqq 4 \gamma\left\|u_{\varepsilon}-u_{\eta}\right\|_{1} e^{\gamma\left\|u_{0}\right\|_{1} t}\left\|u_{0}\right\|_{2}+\left\|f_{\varepsilon}\left(u_{\eta}\right)-f_{\eta}\left(u_{\eta}\right)\right\|_{1}
\end{aligned}
$$

where we used (iii) of Lemma 7 and Theorem II.

\footnotetext{
5 For a sketch of the proof see the appendix.
} 
By Lemma 8 we obtain

$\frac{d}{d t}\left\|u_{\varepsilon}-u_{\eta}\right\|_{1} \leqq 4 \gamma\left\|u_{\varepsilon}-u_{\eta}\right\|_{1} e^{\gamma\left\|u_{0}\right\|_{1} t}\left\|u_{0}\right\|_{2}+4 \gamma|\varepsilon-\eta|\left\|u_{0}\right\|_{1} e^{\gamma\left\|u_{0}\right\|_{1} t}\left\|u_{0}\right\|_{2}$.

Set $\sigma_{\varepsilon, \eta}=4 \gamma T|\varepsilon-\eta|\left\|u_{0}\right\|_{1}\left\|u_{0}\right\|_{2} e^{\gamma\left\|u_{0}\right\|_{1} T}$; we have $\lim _{\varepsilon, \eta \rightarrow 0} \sigma_{\varepsilon, \eta}=0$ and by Gronwall's lemma

$$
\left\|u_{\varepsilon}(t)-u_{\eta}(t)\right\|_{1} \leqq \sigma_{\varepsilon, \eta} e^{4 \gamma\left\|u_{0}\right\|_{2} T e^{\gamma\left\|u_{0}\right\|_{1} T}} .
$$

The proof is complete.

Lemma 11. Let $u_{\varepsilon}$ be the solution of $\left(\mathrm{I}_{\varepsilon}\right)$ and $u=\lim _{\varepsilon \rightarrow 0} u_{\varepsilon}$ the function given in Lemma 10. Then $\lim _{\varepsilon \rightarrow 0} f_{\varepsilon}\left(u_{\varepsilon}\right)=f_{0}(u)$ in $C\left(0, T ; X_{0}\right)$.

Proof. We have $u(t) \in Q \cap X_{1}$ for each $t \in[0, T]$ and:

$$
\begin{aligned}
\left\|f_{\varepsilon}\left(u_{\varepsilon}\right)-f_{0}(u)\right\|_{0} & \leqq\left\|f_{\varepsilon}\left(u_{\varepsilon}\right)-f_{0}\left(u_{\varepsilon}\right)\right\|_{0}+\left\|f_{0}\left(u_{\varepsilon}\right)-f_{0}(u)\right\|_{0} \\
& \leqq 4 \gamma \varepsilon\left\|u_{\varepsilon}\right\|_{1}^{2}+\gamma\left\|u_{\varepsilon}-u\right\|_{1} \\
& \leqq 4 \gamma \varepsilon\left\|u_{0}\right\|_{1}^{2}+\gamma\left\|u_{\varepsilon}-u\right\|_{1},
\end{aligned}
$$

where we used (i) of Lemma 8, Theorem II and Lemma 9. The conclusion follows from Lemma 10.

We can now prove the following theorem:

Theorem III. Assume that $u_{0} \in Q \cap X_{2}$; then for each $T>0$ the problem $\left(\mathrm{I}_{0}\right)$ has a solution $u \in C^{1}\left(0, T ; X_{0}\right)$. Moreover for each $t \in[0, T]$ we have $u(t) \in Q$ and :

$$
\|u(t)\|_{1}=\left\|u_{0}\right\|_{1}, \quad\|u(t)\|_{2} \leqq e^{\gamma\left\|u_{0}\right\|_{1} t}\left\|u_{0}\right\|_{2} .
$$

Proof. Let $u_{\varepsilon}$ be the solution of $\left(\mathrm{I}_{\varepsilon}\right)$; by Lemmas 10 and 11 we have $\lim _{\varepsilon \rightarrow 0} u_{\varepsilon}=u$ in $C\left(0, T ; X_{0}\right)$ and $\lim _{\varepsilon \rightarrow 0} f_{\varepsilon}\left(u_{\varepsilon}\right)=f_{0}(u)$ in $C\left(0, T ; X_{0}\right)$; thus $\lim _{\varepsilon \rightarrow 0} \frac{d u_{\varepsilon}}{d t}=\lim _{\varepsilon \rightarrow 0} f_{\varepsilon}\left(u_{\varepsilon}\right)=f_{0}(u)$. This implies $u \in C^{1}\left(0, T, X_{0}\right)$ and $\frac{d u}{d t}=f_{0}(u)$. Moreover we have $u(0)=\lim _{\varepsilon \rightarrow 0} u_{\varepsilon}(0)=u_{0}$ and $\|u(t)\|_{1}=\lim _{\varepsilon \rightarrow 0}\left\|u_{\varepsilon}(t)\right\|_{1}=\left\|u_{0}\right\|_{1}$.

To prove the second estimate we use Fatou's lemma, $\left\|u_{\varepsilon}(t)\right\|_{2}$ $\leqq e^{\gamma\left\|u_{0}\right\|_{1} t}\left\|u_{0}\right\|_{2}$ and $\lim _{\varepsilon \rightarrow 0} u_{\varepsilon}=u$ in $C\left(0, T ; X_{1}\right)$ to conclude $\|u(t)\|_{2} \leqq e^{\gamma\left\|u_{0}\right\|_{1} t}$ $\left\|u_{1}\right\|_{2}$.

Lemma 12. Let $u_{0}, v_{0} \in Q \cap X_{2}$; if $u \in C^{1}\left(0, T, X_{0}\right)$ (respectively $v \in C^{1}\left(0, T ; X_{0}\right)$ ) is a solution of problem $\left(\mathrm{I}_{0}\right)$ with initial datum $u_{0}$ (respectively $\left.v_{0}\right)$ and $u(t) \in Q(v(t) \in Q)$ for each $t \in[0, T]$, then

$$
\begin{array}{rlrl}
\text { (i) } & \|u(t)\|_{1} & =\left\|u_{0}\right\|_{1}, & \|v(t)\|_{1}=\left\|v_{0}\right\|_{1}, \\
\text { (ii) } & \|u(t)\|_{2} \leqq e^{\gamma t\left\|u_{0}\right\|_{1}}\left\|u_{0}\right\|_{2}, & \|v(t)\|_{2} \leqq e^{\gamma t\left\|v_{0}\right\|_{1}}\left\|v_{0}\right\|_{2}, \\
\text { (iii) } & \|u(t)-v(t)\|_{1} \leqq\left\|u_{0}-v_{0}\right\|_{1} e^{2 \gamma t\left[\left\|u_{0}\right\|_{2} e^{\gamma t t\left\|u_{0}\right\|_{1}}+\left\|v_{0}\right\|_{2} e^{\gamma t\left\|v_{0}\right\|_{1}}\right]}
\end{array}
$$

for each $t \in[0, T]$. 
Proof. It follows from (6) and Lemma 3 that (i) and (ii) of Lemma 7 are formally satisfied by $f_{0}$; thus the assertions (i) and (ii) have the same proofs of Theorem II.

Concerning (iii) we have for a.e. $t \in[0, T]$ :

$$
\frac{d}{d t}\|u-v\|_{1}=\left\langle f_{0}(u)-f_{0}(v), \operatorname{sgn}(u-v)\right\rangle_{1} \leqq 2 \gamma\|u+v\|_{2}\|u-v\|_{1}
$$

where we used Lemma 1 and (iii) of Lemmà 7.

Now

$$
\|u(0)-v(0)\|_{1}=\left\|u_{0}-v_{0}\right\|_{1}
$$

thus the conclusion follows from (i), (ii) and Gronwall's lemma.

As a consequence of Lemma 12 we get the following result:

Theorem IV. Let $u_{0} \in Q \cap X_{2}$, then for each $T>0$ the problem $\left(\mathrm{I}_{0}\right)$ has a unique solution.

Combining Theorems III and IV we obtain an existence and uniqueness theorem for problem (I).

Theorem V. Assume that $u_{0}$ satisfies the following hypothesis

$$
u_{0}(\xi) \geqq 0 \text { a.e., } \quad \int\left(1+\xi^{2}\right)^{2} u_{0} d \xi<+\infty .
$$

Then for each $T>0$ there exists a unique $u:[0, T] \rightarrow L^{1}\left(\mathbb{R}^{3}\right), u(t, \xi) \geqq 0$ a.e. $\xi \in \mathbb{R}^{3}$ for each $t \in[0, T]$, solution of problem (I). Moreover we have

$$
\begin{aligned}
& \int\left(1+\xi^{2}\right) u(t, \xi) d \xi=\int\left(1+\xi^{2}\right) u_{0}(\xi) d \xi \\
& \int\left(1+\xi^{2}\right)^{2} u(t, \xi) d \xi \leqq \int\left(1+\xi^{2}\right)^{2} u_{0}(\xi) d \xi e^{\gamma t \int\left(1+\xi^{2}\right) u_{0} d \xi} \forall t \in[0, T]
\end{aligned}
$$

\section{Appendix}

For the completeness of our work we shall give an outline of the proof of Theorem I. This result is obtained in a more general case in [4] and requires the results of [7]. Two lemmas precede the main arguments.

Lemma 13. Set $Q_{r}=\left\{u \in Q,\|u\|_{0} \leqq r\right\}$; for each $\varepsilon \in(0,1]$ there exists $N_{\varepsilon}(r)$ such that

$$
\left\|f_{\varepsilon}(u)-f_{\varepsilon}(v)\right\|_{0} \leqq N_{\varepsilon}(r)\|u-v\|_{0}, \quad \forall u, v \in Q_{r} .
$$

Proof. The estimate (9) is an immediate consequence of assumption (H) of Section 3.

Lemma 14. For each $r>0$ there exists $n(\varepsilon, r)$ such that the following equation

$$
n u-f_{\varepsilon}(u)=v, \quad \varepsilon \in(0,1], \quad v \in Q_{r}
$$

has a unique solution $u \in Q_{r}$ for each $n \geqq n(\varepsilon, r)$. 
Proof. Set

$$
\begin{aligned}
u_{1} & =\frac{v}{n} \\
\ldots \ldots \ldots \ldots \ldots \ldots \ldots \ldots \ldots \ldots & v+f_{\varepsilon}^{+}\left(u_{k}\right) \\
u_{k+1} & =\frac{1}{n+\frac{1}{u_{k}} f_{\varepsilon}^{-}\left(u_{k}\right)} .
\end{aligned}
$$

It is easy to see that $u_{k} \in Q_{r}$ and that for $n \geqq n(\varepsilon, r)$ there exists $\lim _{k \rightarrow \infty} u_{k}=u \in Q_{r}$. As $f_{\varepsilon}$ is locally lipschitz continuous the conclusion follows.

By Lemma 14 we can define the "local resolvent" function in the following way:

$$
R_{Q_{r}}\left(n, f_{\varepsilon}\right)=\left(n-f_{\varepsilon}\right)^{-1}: Q_{r} \rightarrow Q_{r}, \quad n \geqq n(\varepsilon, r)
$$

it is not difficult to see that

$$
\begin{array}{r}
\left\|R_{Q_{r}}\left(n, f_{\varepsilon}\right) u\right\|_{0} \leqq \frac{1}{n}\|u\|_{0}, \quad \forall u \in Q_{r}, \\
\left\|R_{Q_{n r}}\left(n, f_{\varepsilon}\right) n u-R_{Q_{n r}}\left(n, f_{\varepsilon}\right) n v\right\|_{0} \leqq \frac{n}{n-N_{\varepsilon}(r)}\|u-v\|_{0}
\end{array}
$$

for each $u, v \in Q_{r}$ and $n \geqq \max \left\{n(\varepsilon, r), N_{\varepsilon}(r)\right\}$.

For each fixed $r>0$ and any integer $n \geqq n(\varepsilon, r)$ we define the Yosida's approximation function

$$
f_{\varepsilon, n}^{r}=n R_{Q_{n r}}\left(n, f_{\varepsilon}\right) n-n=f_{\varepsilon} R_{Q_{n r}}\left(n, f_{\varepsilon}\right) n .
$$

From the local lipschitz continuity of $f_{\varepsilon}$ for each $r>0$ we have

$$
\lim _{n \rightarrow \infty} f_{\varepsilon, n}^{r}(u)=f_{\varepsilon}(u)
$$

uniformly in $Q_{r}$.

Now consider the integral equation

$$
\left(\mathrm{I}_{n}\right) \quad u_{n}(t)=u_{0}+\int_{0}^{t} f_{\varepsilon, n}^{r}\left(u_{n}\right)(s) d s, \quad t \in[0, T], u_{0} \in Q_{r} .
$$

The following lemma holds:

Lemma 15. For each $u_{0} \in Q_{r}$ and $n>\max \left\{n(\varepsilon, r), N_{\varepsilon}(r)\right\}$ there exists a solution $u_{n}(t)$ of problem $\left(\mathrm{I}_{n}\right)$ such that $\left\|u_{n}(t)\right\|_{0} \leqq\left\|u_{0}\right\|_{0}$ for each $t \in[0, T]$.

Proof. Set $Y=C\left(0, T ; X_{0}\right), K=\left\{u \in Y, u(t) \in Q_{\left\|u_{0}\right\|_{0}}\right.$ for $\left.t \in[0, T]\right\}$ and consider the function $\tau_{n}: K \rightarrow Y$ defined by

$$
\left(\tau_{n}(u)\right)(t)=\exp (-n t) u_{0}+n \int_{0}^{t} \exp (-n(t-s)) R_{Q_{n} r}\left(n, f_{\varepsilon}\right) n u(s) d s .
$$


Using (i) and (ii) it is not difficult to see that $\tau_{n}(K) \subset K$ and that for $T=T_{0}$ sufficiently small (depending on $r$ and $n$ ) $\tau_{n}$ is a contraction which maps $K$ into itself. Consequently there is a function $u_{n} \in K$ such that $\tau_{n} u_{n}=u_{n}$ and it is easy to see that $u_{n}$ satisfies $\left(\mathrm{I}_{n}\right)$. Moreover as we have $\left\|u_{n}\left(T_{0}\right)\right\|_{0} \leqq\left\|u_{0}\right\|_{0}$ we can consider the same problem $\left(\mathrm{I}_{n}\right)$ with the initial point $u_{0}$ replaced by $u_{n}\left(T_{0}\right)$ and this allows us to extend to the interval $\left[0,2 T_{0}\right]$ the solution of $\left(\mathrm{I}_{n}\right)$ with initial point $u_{0}$. Iterating this argument we extend the solution of $\left(\mathrm{I}_{n}\right)$ to $[0, T]$ with $T$ arbitrarily given; obviously we have $\left\|u_{n}(t)\right\|_{0} \leqq\left\|u_{0}\right\|_{0}$ for $t \in[0, T]$.

From (ii) and Gronwall's lemma it follows that there exists $u \in K$ such that $\lim _{n \rightarrow \infty} u_{n}=u$ in $Y$; thus, using (iii), we obtain:

$$
\text { (I) } u(t)=u_{0}+\int_{0}^{t} f_{\varepsilon}(u)(s) d s, \quad t \in[0, T] ; \varepsilon \in(0,1] \text {. }
$$

Finally the uniqueness of the solution of problem (I) and its differentiability are a straightforward consequence of the local lipschitz continuity of $f_{\varepsilon}$.

The author thanks Profs. G. Da Prato and G. Gallavotti for stimulating discussions.

\section{References}

1. Bodmer, R.: Zur Boltzmanngleichung. Zürich: Seminar für Theoretische Physik, E.T.H.

2. Cercignani, C.: Mathematical methods in kinetic theory. New York: Plenum Press 1969

3. Da Prato,G.: Somme d'applications non linéaires. In: Symposia Mathematica vol. VII. Roma: Istituto Nazionale di Alta Matematica 1971

4. Di Blasio,G.: Strong solution for Boltzmann equation in the spatially homogeneous case. Boll. Unione Matematica Italiana. 8, 127-136 (1973)

5. Dunford, N., Schwartz, J.T.: Linear operators I. New York: Interscience 1958

6. Grad,H.: Principles of kinetic theory of gases, Handbuch der Physik, V. 12, BerlinGöttingen-Heidelberg: Springer 1958

7. Iannelli, M.: A note on some non-linear non-contraction semigroups. Boll. Unione Matematica Italiana. 6, 1015-1025 (1970)

8. Kato, T.: Non linear semigroups and evolution equations. J. Math. Soc. Japan. 19, $508-520(1967)$

9. Povzner,A.Ja.: The Boltzmann equation in the kinetic theory of gases. Mat. Sbornik 58, 65-86 (1962). Translated in: American Mat. Soc. Translations, Series 2, 47, 193-216 (1965) 